\title{
Clinicopathological Characteristics and Prognosis of Remnant Gastric Cancer
}

\author{
Sang Bong Lee, Jae Hun Kim, Dae Hwan Kim, Tae Yong Jeon, Dong Heon Kim, Gwang Ha Kim¹, and Do Youn Park \\ Department of Surgery, Postgraduate School of Medicine, Pusan National University, Busan, Korea, ${ }^{1}$ Internal Medicine, Postgraduate School of \\ Medicine, Pusan National University, Busan, Korea, ${ }^{2}$ Pathology, Postgraduate School of Medicine, Pusan National University, Busan, Korea
}

Purpose: The long-term survival rate of gastric cancer patients after surgery has recently increased as a result of making an early diagnosis of gastric cancer. Therefore, the incidence of remnant gastric cancer is increasing. This study was performed to evaluate the clinicopathological characteristics and prognosis of patients with remnant gastric cancer.

Materials and Methods: From January 2005 to December 2009, twenty-nine patients with remnant gastric cancer and who underwent surgery at Pusan National University Hospital were enrolled in this study. We retrospectively reviewed and analyzed their medical records. We also divided them into two groups: the remnant gastric cancer (RGC)-B group (first operation for benign disease) and the RGC-M group (first operation for malignant disease).

Results: The RGC-B group included ten patients and the RGC-M group included nineteen patients. The mean interval between the first and second operations was 17 years. The curative resection rate was 93.1\% (27/29). The postoperative complication rate was $20.7 \%$ (6/29) and there was no perioperative mortality. Ten (37\%) of twenty-seven patients experienced recurrence after curative resection and eight patients (27.6\%) expired due to aggravation of remnant stomach cancer. An advanced TNM stage and non-curative resection were the negative prognostic factors for survival for patients with remnant stomach cancer $(P=0.0453$ and $P<0.001)$. The RGC-M group showed a shorter interval $(P<0.001)$ and the RGC-B group had more advanced TNM stage $(P=0.003)$.

Conclusions: Long-term follow-up should be considered not only for patients who undergo an operation for malignant disease, but also for the patients who underwent an operation for benign disease. When remnant gastric cancer is diagnosed, curative resection is essential to improve the survival.

Key Words: Gastric remnant cancer, Prognosis, Survival

\section{Introduction}

Remnant gastric cancer is generally defined as cancer that has developed in the remnant stomach 5 years after gastrectomy. However, in many recent reports, remnant gastric cancer has been defined as cancer that has developed in the remnant stomach after partial gastrectomy regardless of the disease or the operative

Correspondence to: Dong Heon Kim

Department of Surgery, Pusan National University School of Medicine,

305, Gudeok-ro, Seo-gu, Busan 602-739, Korea

Tel: +82-51-240-7238, Fax: +82-51-247-1365

E-mail: kdhun@pusan.ac.kr

Received August 27, 2010

Accepted Octorber 14, 2010

This work was supported for two years by Pusan National University Research Grant. methods used for gastrectomy. $(1,2)$ With the development of the medication for treating gastric ulcer, partial gastrectomy for benign disease is on the decrease, and so remnant gastric carcinoma that develops after surgery for benign diseass is also on the decrease. Nonetheless, on account of performing regular endoscopic examination, the rate of detecting early gastric cancer has recently increased, and so the long-term survival after surgery has increased. As a consequence, remnant gastric cancer after partial gastrectomy for malignant diseases is on the increase. In recent studies, the incidence of remnant gastric cancer that developed after distal gastrectomy for gastric cancer has been reported to be $1 \sim 2 \%$ of all the gastric cancer patients. $(1,3)$

It has been reported that for patients with remnant gastric cancer, there is a high stage of disease at the time of diagnosis, lymph 
node metastasis is abundant and the rate of curative resection is low because of the frequent invasion to adjacent organs and so prognosis is poor. $(3,4)$ Therefore, it is very important to understand the characteristics of remnant gastric cancer and to determine its prognostic factors in order to decide on the treatment methods. There are only a small number of studies that have reported on remnant gastric cancer.

We studied the clinicopathological characteristic of patients who underwent surgery for remnant gastric cancer, and we suggest a treatment strategy based on the results.

\section{Materials and Methods}

The study was conducted on 29 patients who were diagnosed as having remnant gastric cancer at our Department of Surgery, Pusan University Hospital, from January 2005 to December 2009. The preoperative data of the patients (age, gender, the disease at the time of the first operation, the operative methods at the time of the first operation, the interval from the first operation to the diagnosis of remnant gastric cancer), the operative data (the operative methods for remnant gastric cancer, whether radical resection was performed, whether combined resection was performed at the time of operation, operation time, whether transfusion was performed, and hospitalization period) and the postoperative data (the location of the remnant gastric cancer, the histological classification, size and TNM stage, the presence or absence of lymphovascular invasion, the presence or absence of perineral invasion, the follow-up period, recurrence and death) were analyzed retrospectively.

In regard to surgery, after abdominal incision, total gastrectomy with lymphadectomy more than D2 and Roux-en-Y anastomosis were performed in all the patients. At the time of lymphadenectomy, lymphadenectomy for the 19th lymph node station (the infradiaphragmatic nodes) and the 20th lymph node station (the esophageal hiatal nodes) was included for all the patients. Except for the patients with suspected metastasis according to the preoperative examinations or that was macroscopically determined during surgery, lymphadenectomy for the 13th lymph node station (the retropancreatic nodes) and the 16th lymph node station (the paraaortic nodes) was not performed. For the patients who underwent Billroth-I anstomosis during their previous surgeries, the duodenum was dissected from the pancreas and subsequently a portion of the duodenum was resected, and lymphadenectomy for the 17th lymph node station (the anterior pancreatic nodes) was performed. For the patients who underwent Billroth II anastomosis and Roux-en-Y anastomosis, from the anastomotic site, at least 10 $\mathrm{cm}$ the ileum was resected together with the ileal mesenteric lymph nodes. For the patients whose remnant gastric cancer was located near the esophagogastric junction, the esophaseal hitus was incised and lymphadenectomy for the 110th (lower paraesophageal nodes) and the 111th lymph node (supradiaphragmatic nodes) stations was performed.

The location of remnant gastric cancer was divided to invasion to the anastomotic site, locations other than the anastomotic site, and the entire remnant stomach. The TNM stage was classified according to the American Joint Committee on Cancer (AJCC), 7th edition. The histological classification was classified to the differentiated group (papillary, well differentiated and moderately differentiated carcinoma) and the undifferentiated group (poorly differentiated, mucinous and signet ring cell carcinoma). According to Lauren's classification, the cancers were classified to the intestinal type and the diffuse type. In addition, in our study, according to the disease at the time of the first operation, the patients were divided to the patient group who underwent partial gastrectomy for benign diseases (RGC-B) and the patient group who underwent partial gastrectomy for malignant diseases ( $R G C-M)$, and their clinicopathological characteristics were compared and analyzed. In addition, the factors affecting the survival rate of all the patients were analyzed.

SPSS for Windows (ver. 12.0, SPSS Inc., Chicago. IL. USA) was applied for statistical analysis. For the comparison of the RGC-B and the RGC-M, the Mann-Whitney U-test and Fisher's exact test were performed. Kaplan-Meier Methods were applied for the analysis of the survival rate. $\mathrm{P}-$ values $<0.05$ were considered to be statistically significant.

\section{Results}

\section{The clinicopathological characteristics of the total patients}

The clinicopathological characteristics of all 29 patients who underwent surgery for remnant gastric cancer at our hospital are shown in Table 1. The clinicopathological characteristics of the 10 recurrent patients of the 27 patients who underwent curative resection are shown in Table 2. The RGC-B patient group was 10 patients, and the RGC-M patient group was 19 patients. Twenty seven patients $(93.1 \%)$ received curative resection and 2 patients received only palliative resection because of intraperitoneal metastasis. An average of 21 lymph nodes were retrieved for postsurgical histo- 
Table 1. Clinicopathological characteristics of the 29 patients with RGC

\begin{tabular}{llc}
\hline \multicolumn{1}{c}{ Total number } & & $\mathrm{N}=29$ \\
\hline Age (years) & Mean \pm S.D. & $61 \pm 10$ \\
Sex & Male & 25 \\
& Female & 14 \\
Interval $^{*}$ (years) & Mean \pm S.D. & $17 \pm 14.5$ \\
Curative resection (\%) & $(+)$ & $27(93.1 \%)$ \\
& $(-)$ & $2(6.9 \%)$ \\
Retrieved lymph node & Mean \pm S.D. & $21 \pm 10$ \\
Combined resection (\%) & & $12(41.4 \%)$ \\
Operation time (min) & Mean \pm S.D. & $248 \pm 93$ \\
Hospital stay (days) & Mean \pm S.D. & $19 \pm 16$ \\
Postoperative morbidity (\%) & & $6(20.7 \%)$ \\
Tumor size (mm) & Mean \pm S.D. & $46 \pm 36$ \\
TNM stage (\%) & I & $11(37.9 \%)$ \\
& II & $2(6.9 \%)$ \\
Follow-up period (months) & Mean \pm S.D. & $14(48.3 \%)$ \\
Death (\%) & III & $2(6.9 \%)$ \\
\hline & IV & $24 \pm 17$ \\
& & $8(27.6 \%)$ \\
\hline (II & & \\
& &
\end{tabular}

RGC = remnant gastric cancer; S.D. = standard deviation. ${ }^{\star}$ Interval between initial operation to diagnosis.

logical tests. Combined resection was performed in 12 patients. For D2 lymphadenectomy, splenectomy was performed on 6 patients. In 4 patients, invasion to the transverse colon was macroscopically suspected and so segmental resection was performed. Nonetheless, only 2 patients showed invasion on the histology exam. In addition, hepatic invasion was suspected in 2 patients and so hepatic wedge resection was performed, yet invasion was not detected by the histological exam. Postoperative complications were detected in 6 patients $(20.7 \%)$. Four patients developed postoperative ileus and 2 patients developed leakage from the anastomotic site, yet none of these patients died. The average follow-up period of the patients was $24 \pm 17$ months. During the follow-up period, the number of patients who recurred after radical resection were 10 patients of the 27 patients $(37 \%)$. Among the entire 29 patients, 8 patients died (27.6\%).

\section{The clinicopathological characteristics according} to the disease at the time of the first operation

When comparing the RGC-B patient group and the RGC-M patient group, gender, the method of anastomosis at the first op-
Table 2. Clinicopathological characteristics of recurrence after curative resection

\begin{tabular}{llcc}
\hline & & $\begin{array}{c}\text { Benign } \\
(\mathrm{N}=8)\end{array}$ & $\begin{array}{c}\text { Malignant } \\
(\mathrm{N}=19)\end{array}$ \\
\hline Recur & $\mathrm{N}(\%)$ & $2(25 \%)$ & $8(44.1 \%)$ \\
Site of recurrence & Liver & 0 & 2 \\
& Lung & 0 & 2 \\
& Peritoneum & 0 & 4 \\
Stage & Anastomotic site & 2 & 0 \\
& I & 0 & 2 \\
& II & 0 & 0 \\
& III & 2 & 6 \\
\hline
\end{tabular}

eration, the tumor location, whether transfusion was performed, whether radical resection was performed, the histological classification, the tumor size, recurrence and death did not show significant differences between the 2 groups. The age of the RGC-B patient group was higher than that of the RGC-M group ( $66 \pm 8$ years vs. $58 \pm 10$ years, respectively, $\mathrm{P}=0.023)$. It was observed that the interval from the first operation to the diagnosis of remnant gastric cancer for the RGC-B group was longer than that of the RGC-M patient group ( $36 \pm 6$ years vs. $7 \pm 5$ years, respectively, $\mathrm{P}<0.001$ ). The $\mathrm{T}$ stage of the RGC-B patient group was higher than that of the RGC-M group $(\mathrm{P}=0.003)$. The $\mathrm{N}$ stage of the $\mathrm{RGC}-\mathrm{B}$ patient group was higher than that of the RGC-M patient group $(\mathrm{P}<0.001)$. In addition, more lymphovascular invasion as well as perineural invasion were observed in the $\mathrm{RGC}-\mathrm{B}$ patient group $(\mathrm{P}=0.021$ and $\mathrm{P}=0.005$, respectively). In regard to the TNM stage, similarly, the RGC-B patient group showed more advanced stages $(\mathrm{P}=0.003)$, yet the number of $\mathrm{M}$ stage patients of the two groups did not show a difference (Table 3).

\section{Factors affecting the survival of the total pa- tients}

The patient age and gender, the initial gastric disease at the time of the first operation, the anastomosis method at the first operation and the interval from the first operation to the diagnosis of remnant gastric cancer did not affect the survival rate. In addition, the location of tumor in the remnant stomach, whether or not transfusion was performed during the operation and the histologic classification did not affect the survival rate. The $\mathrm{N}$ stage, lymphovascular invasion and perineural invasion between the RGC-B group and the RGC-M groups showed statistically significant differences, but 
Lee SB, et al.

Table 3. Clinicopathological characteristics according to the initial gastric disease

\begin{tabular}{|c|c|c|c|c|}
\hline \multicolumn{2}{|c|}{ Factor } & \multirow{2}{*}{$\frac{\text { Benign }(\mathrm{N}=10)}{66 \pm 8}$} & \multirow{2}{*}{$\begin{array}{c}\text { Malignancy }(\mathrm{N}=19) \\
58 \pm 10\end{array}$} & \multirow{2}{*}{$\frac{\text { P-value }^{\dagger}}{0.023}$} \\
\hline Age (years) & Mean \pm S.D. & & & \\
\hline Sex & Male/Female & $8 / 2$ & $17 / 2$ & 0.592 \\
\hline \multirow{3}{*}{$\begin{array}{l}\text { Reconstruction } \\
\text { of initial operation }\end{array}$} & B-I & 2 & 5 & 0.410 \\
\hline & B-II & 8 & 12 & \\
\hline & Roux-en-Y & 0 & 4 & \\
\hline Interval ${ }^{\star}$ (years) & Mean \pm S.D. & $36 \pm 6$ & $7 \pm 5$ & $<0.001$ \\
\hline \multirow[t]{3}{*}{ Tumor location } & Anastomotic site & 4 & 8 & 0.322 \\
\hline & Non-anastomotic site & 6 & 7 & \\
\hline & Entire & 0 & 4 & \\
\hline Transfusion & $(+) /(-)$ & $6 / 4$ & $6 / 13$ & 0.236 \\
\hline Curative resection & $(+) /(-)$ & $8 / 2$ & $19 / 0$ & 0.111 \\
\hline T stage & $1 / 2,3,4$ & $0 / 10$ & $11 / 8$ & 0.003 \\
\hline $\mathrm{N}$ stage & $(+) /(-)$ & $10 / 0$ & $2 / 17$ & $<0.001$ \\
\hline M stage & $(+) /(-)$ & $2 / 8$ & $0 / 19$ & 0.111 \\
\hline TNM stage & I / II, III, IV & $0 / 10$ & $11 / 8$ & 0.003 \\
\hline \multirow[t]{4}{*}{ Histology } & Differentiated & 4 & 13 & 0.236 \\
\hline & Undifferentiated & 6 & 6 & \\
\hline & Intestinal & 4 & 11 & 0.450 \\
\hline & Diffuse & 6 & 8 & \\
\hline Lymphovascular invasion & $(+) /(-)$ & $8 / 2$ & $6 / 13$ & 0.021 \\
\hline Perineural invasion & $(+) /(-)$ & $8 / 2$ & $4 / 15$ & 0.005 \\
\hline Tumor size (mm) & Mean \pm S.D. & $46 \pm 10$ & $45 \pm 19$ & 0.394 \\
\hline Recur & $(+) /(-)$ & $2 / 6^{\dagger}$ & $8 / 11$ & 0.666 \\
\hline Death & $(+) /(-)$ & $2 / 8$ & $6 / 13$ & 0.675 \\
\hline
\end{tabular}

S.D. $=$ standard deviation. ${ }^{*}$ Interval between initial operation to diagnosis; ${ }^{\dagger} \mathrm{n}=8$.

Table 4. Univariate analysis of the factors affecting the survival (preoperative data)

\begin{tabular}{|c|c|c|c|c|}
\hline \multicolumn{2}{|c|}{ Factor } & \multirow{2}{*}{$\begin{array}{c}\begin{array}{c}\text { Total } \\
(\mathrm{N})\end{array} \\
15\end{array}$} & \multirow{2}{*}{$\begin{array}{c}\text { Death } \\
(\mathrm{N})\end{array}$} & \multirow{2}{*}{$\begin{array}{l}\text { P-value } \\
0.9304\end{array}$} \\
\hline Age (years) & $<60$ & & & \\
\hline & $>60$ & 14 & 4 & \\
\hline \multirow[t]{2}{*}{ Sex } & Male & 25 & 8 & 0.3931 \\
\hline & Female & 4 & 0 & \\
\hline \multirow[t]{2}{*}{ Initial gastric disease } & Benign & 10 & 2 & 0.5581 \\
\hline & Malignancy & 19 & 6 & \\
\hline \multirow{3}{*}{$\begin{array}{l}\text { Reconstruction } \\
\text { of initial operation }\end{array}$} & B-I & 5 & 2 & 0.1010 \\
\hline & B-II & 20 & 2 & \\
\hline & Roux-en-Y & 4 & 4 & \\
\hline \multirow[t]{2}{*}{ Interval $^{\star}$ (years) } & $\leq 15$ & 18 & 6 & 0.4885 \\
\hline & $\geq 15$ & 11 & 2 & \\
\hline
\end{tabular}

${ }^{\star}$ Interval between initial operation to diagnosis. the survival rates did not show a difference. Among the 27 patients who underwent curative resection, 6 patients (22.2\%) died. In the patient group that underwent palliative resection, 2 patients (100\%) died, and a statistically significant difference was shown between the group who underwent curative resection and the group who underwent pallative resection $(\mathrm{P}<0.001)$. Similarly, on comparing the mortality (of the T stage I and II patients (15.4\%), the stage III patients (16.7\%) and the IV patients (100\%), the higher grade patients showed higher mortality, and a significant difference was seen ( $\mathrm{P}$ $<0.001)$ ). (In regard to tumor size, the survival rate between tumors larger than $5 \mathrm{~cm}$ and less than $5 \mathrm{~cm}$ showed a statistically difference $(\mathrm{P}<0.001))$. In regard to the tumor size, based on $5 \mathrm{~cm}$, the survival rate showed a statistically significant difference $(\mathrm{P}<0.001)$. However, when multivariate analysis was performed on the factors that were statistically significant on the univariate analysis, all the factors were not statistically significant (Table 4, 5). 
Table 5. Univariate analysis of the factors affecting the survival (post-operation data)

\begin{tabular}{|c|c|c|c|c|}
\hline \multicolumn{2}{|c|}{ Factor } & $\begin{array}{l}\text { Total } \\
(\mathrm{N})\end{array}$ & $\begin{array}{c}\text { Death } \\
(\mathrm{N})\end{array}$ & $\begin{array}{c}\mathrm{P}- \\
\text { value }\end{array}$ \\
\hline \multirow[t]{3}{*}{ Tumor location } & Anastomotic site & 13 & 4 & \multirow[t]{3}{*}{0.7623} \\
\hline & Non-anastomotic site & 12 & 4 & \\
\hline & Entire & 4 & 0 & \\
\hline \multirow[t]{2}{*}{ Transfusion } & $(+)$ & 12 & 4 & \multirow[t]{2}{*}{0.8498} \\
\hline & $(-)$ & 17 & 4 & \\
\hline \multirow[t]{2}{*}{ Curative resection } & $(+)$ & 27 & 6 & \multirow[t]{2}{*}{$<0.001$} \\
\hline & $(-)$ & 2 & 2 & \\
\hline \multirow[t]{3}{*}{ T stage } & I, II & 13 & 2 & \multirow[t]{3}{*}{$<0.001$} \\
\hline & III & 12 & 2 & \\
\hline & IV & 4 & 4 & \\
\hline \multirow[t]{2}{*}{ N stage } & $(+)$ & 12 & 4 & \multirow[t]{2}{*}{0.8571} \\
\hline & $(-)$ & 17 & 4 & \\
\hline \multirow[t]{2}{*}{ M stage } & $(+)$ & 2 & 2 & \multirow[t]{2}{*}{$<0.001$} \\
\hline & $(-)$ & 27 & 6 & \\
\hline \multirow[t]{2}{*}{ TNM stage } & I, II & 15 & 2 & \multirow[t]{2}{*}{0.0453} \\
\hline & III, IV & 14 & 6 & \\
\hline \multirow[t]{4}{*}{ Histology } & Differentiated & 17 & 4 & \multirow[t]{2}{*}{0.1669} \\
\hline & Undifferentiated & 12 & 4 & \\
\hline & Intestinal & 15 & 4 & \multirow[t]{2}{*}{0.2072} \\
\hline & Diffuse & 14 & 4 & \\
\hline \multirow{2}{*}{$\begin{array}{l}\text { Lymphovascular } \\
\text { invasion }\end{array}$} & $(+)$ & 14 & 6 & \multirow[t]{2}{*}{0.1149} \\
\hline & $(-)$ & 15 & 2 & \\
\hline \multirow{2}{*}{$\begin{array}{l}\text { Perineural } \\
\text { invasion }\end{array}$} & $(+)$ & 12 & 6 & \multirow[t]{2}{*}{0.0917} \\
\hline & $(-)$ & 17 & 2 & \\
\hline \multirow[t]{2}{*}{ Tumor size $(\mathrm{cm})$} & $\leq 5$ & 23 & 4 & \multirow[t]{2}{*}{$<0.001$} \\
\hline & $>5$ & 6 & 4 & \\
\hline
\end{tabular}

\section{Discussion}

The treatments for remnant gastric cancer include surgical treatment, radiation therapy, chemotherapy and immunological therapy, which are all similar to that used for primary gastric cancer. Nonetheless, surgery is known to be the only curative method. Generally, the surgical treatments include the complete resection of the remnant stomach and more than D2 lymphadenectomy.(5) In several reports, the rates of performing radical resection for remnant gastric cancer were diverse from 50 90\%.(1,6,7) In our study, among the 29 patients, 27 patients $(93.1 \%)$ received total gastrectomy with more than D2 lymphadenectomy, and the rate was slightly higher than that of other studies. However, such results have the possibility of a selection bias by including only the patients for whom radical resection could be performed among all the patients diagnosed with remnant gastric carcinoma. Hu et al.(5) have reported that at the time of surgery for the remnant stomach cancer, the rate of the combined resection of adjacent organs could be increased because of the change of the pattern of lymph node metastasis due to previous surgeries and the adhesion of the adjacent organs. In other reports, similarly, the rate of the combined resection of adjacent organs was reported as high as 40 70\%.(8,9) We performed combined resection of adjacent organs in $41.4 \%$ of the cases, and similar findings were seen.

The interval from the first operation to the diagnosis of remnant gastric cancer was very different depending on the studies. This has generally been reported to be 22 27 years for the RGC-B patient group and that of the RGC-M group has been reported to be approximately 8.3 9 years. $(1,2,10)$ In our study, similarly, the interval from the first operation to the diagnosis of recurrent gastric cancer for the RGC-B patient group was $36 \pm 6$ years, and it was longer than the $7 \pm 5$ years of the RGC-M group. Such a difference is generally considered to be due to the different mechanisms for the development of remnant gastric cancer for the two groups. In the RGC-B patient group, the irritation of the mucosa by the reflux of bile and pancreatic juice from the duodenum is considered to be a major factor for the development of remnant gastric cancer. On the other hand, in the RGC-M patient group, specific precancerous conditions are considered to be factors for the development of remnant gastric cancer. $(1,4,11)$ Therefore, the interval to the development of remnant gastric cancer is longer, and more remnant gastric cancer develops at the anastomotic site in the $\mathrm{RGC}-\mathrm{B}$ patient group than that in the RGC-M patient group. $(1,4,6)$ However, in our study, the location of remnant gastric cancer of the RGC-B patient group and the RGC-M patient group did not show a great difference. Therefore, it is thought that other factors are present besides the irritation of the mucosa by the reflux of bile and pancreatic juice from the duodenum. Kaminishi et al.(12) suggested that due to the weakening of the mucosal defense capacity of the remnant stomach, which is caused by denervation after distal gastrectomy, the development of remnant gastric carcinoma may be increased. In addition, in our study, the average age of the RGC-B patient group was $66 \pm 8$ years, which is the age that is prone to develop gastric cancer, and the possibility that age exerts effects on the development of remnant gastric cancer and so remnant gastric cancer is developed in the area other than the anastomotic site should be 
considered.(13)

In addition, the $\mathrm{RGC}-\mathrm{B}$ patient group and the $\mathrm{RGC}-\mathrm{M}$ group showed a difference for the TNM stage. The RGC-B patient group showed a more advanced $\mathrm{T}$ stage as well as $\mathrm{N}$ stage compared to that of RGC-M patient group. This is thought to be due to the fact that regular follow-up such as endoscopy was not commonly performed in the RGC-B patient group, and so more advanced remnant gastric cancer was detected in many of these patients. Yet Kodera et al.(2) stated that in the patient group that underwent distal gastrectomy for malignant diseases, at the time of operation, lymphadenectomy was performed radically, and so lymph node metastasis would be relatively lower than that in the patient group that underwent operation for benign diseases.

It has been reported that the prognosis of patients with remnant gastric cancer is generally poor and 5-year survival rate is 40 60\%. The follow-up period of our study was an average of 24 \pm 17 months, the disease-free survival rate after curative resection was $62.9 \%$ and the survival rate of the total patients was $72.4 \%$. Although we obtained results similar to those of the previous reports, the follow-up period of our study was short and so the results of the long-term follow-up are required. It is known that the factors affecting survival rate are the TNM stage, whether or not radical resection was performed and tumor size. Hu et al.(5) have reported that the 5-year survival rate of the patient group that received curative resection was higher than that of the group that did not receive curative resection. In addition, in another study, the patient group with a lower TNM stage showed a 77\% survival rate, which was higher than the generally reported 5-year survival rate for patients with remnant gastric cancer.(14) It is thought that the radical resection and low TNM stage resulted from the early detection of remnant gastric cancer, and so regular follow-up after distal gastrectomy may play an important role. Ojima et al.(15) performed a multivariate analysis on the factors that may affect the possibility of curative resection of remnant gastric carcinoma, and they suggested that the regular follow-up is the only factor affecting the possibility of achieving curative resection.

Based on our study, although the number of patients was small, we found that the disease stage as well as radical resection are very important for improving the survival rate of patients with remnant gastric carcinoma. Therefore, appropriate follow-up observation after distal gastrectomy is very important for improving the survival rate of patients with remnant gastric cancer, regardless of the previous disease. After the detection of remnant gastric cancer, aggressive efforts when performing radical resection are required during the operation.

\section{References}

1. Ohashi M, Katai H, Fukagawa T, Gotoda T, Sano T, Sasako M. Cancer of the gastric stump following distal gastrectomy for cancer. Br J Surg 2007;94:92-95.

2. Kodera Y, Yamamura Y, Torii A, Uesaka K, Hirai T, Yasui K, et al. Gastric remnant carcinoma after partial gastrectomy for benign and malignant gastric lesions. J Am Coll Surg 1996;182:1-6.

3. Kaneko K, Kondo H, Saito D, Shirao K, Yamaguchi H, Yokota T, et al. Early gastric stump cancer following distal gastrectomy. Gut 1998;43:342-344.

4. Firat O, Guler A, Sozbilen M, Ersin S, Kaplan H. Gastric remnant cancer: an old problem with novel concerns. Langenbecks Arch Surg 2009;394:93-97.

5. Hu X, Tian DY, Cao L, Yu Y. Progression and prognosis of gastric stump cancer. J Surg Oncol 2009;100:472-476.

6. Ahn HS, Kim JW, Yoo MW, Park do J, Lee HJ, Lee KU, et al. Clinicopathological features and surgical outcomes of patients with remnant gastric cancer after a distal gastrectomy. Ann Surg Oncol 2008;15:1632-1639.

7. Thorban S, Böttcher K, Etter M, Roder JD, Busch R, Siewert JR. Prognostic factors in gastric stump carcinoma. Ann Surg 2000;231:188-194.

8. Lissens P, Filez L, Aerts R, D'Hoore A, Van Cutsem E, Ectors $\mathrm{N}$, et al. Surgery for gastric remnant carcinoma following Billroth II gastrectomy. Eur J Surg Oncol 1997;23:518-521.

9. An JY, Youn HG, Ha TK, Choi MG, Kim KM, Noh JH, et al. Clinical significance of tumor location in remnant gastric cancers developed after partial gastrectomy for primary gastric cancer. J Gastrointest Surg 2008;12:689-694.

10. Sinning C, Schaefer N, Standop J, Hirner A, Wolff M. Gastric stump carcinoma - epidemiology and current concepts in pathogenesis and treatment. Eur J Surg Oncol 2007;33:133139.

11. Furukawa H, Iwanaga T, Hiratsuka M, Imaoka S, Ishikawa O, Kabuto T, et al. Gastric remnant cancer as a metachronous multiple lesion. Br J Surg 1993;80:54-56.

12. Kaminishi M, Shimizu N, Yamaguchi H, Hashimoto M, Sakai $\mathrm{S}$, Oohara T. Different carcinogenesis in the gastric remnant after gastrectomy for gastric cancer. Cancer 1996;77(8 Suppl):1646-1653. 
13. Lee HJ, Yang HK, Ahn YO. Gastric cancer in Korea. Gastric Cancer 2002;5:177-182.

14. Zhang Y, Tokunaga A, Masuda G, Okuda T, Kiyama T, Yoshiyuki T, et al. Surgical treatment of gastric remnant-stump cancer. J Nippon Med Sch 2002;69:489-493.
15. Ojima T, Iwahashi M, Nakamori M, Nakamura M, Naka T, Katsuda M, et al. Clinicopathological characteristics of remnant gastric cancer after a distal gastrectomy. J Gastrointest Surg 2010;14:277-281. 